\title{
Synthesis of 4-Substituted-1,2-Dihydroquinolines by Means of Gold-Catalyzed Intramolecular Hydroarylation Reaction of $N$-Ethoxycarbonyl-N-Propargylanilines
}

\author{
Antonio Arcadi ${ }^{1}(0)$, Andrea Calcaterra ${ }^{2}\left(\mathbb{D}\right.$, Giancarlo Fabrizi ${ }^{2}$, Andrea Fochetti ${ }^{2}$, Antonella Goggiamani ${ }^{2, *}$, \\ Antonia Iazzetti ${ }^{2, *}$, Federico Marrone ${ }^{2}$, Vincenzo Marsicano ${ }^{1}\left[\right.$, Giulia Mazzoccanti ${ }^{2}\left(\mathbb{D}\right.$ and Andrea Serraiocco ${ }^{2}$ \\ 1 Dipartimento di Scienze Fisiche e Chimiche, Università degli Studi di L'Aquila, 67100 Coppito, Italy; \\ antonio.arcadi@univaq.it (A.A.); vincenzo.marsicano@graduate.univaq.it (V.M.) \\ 2 Dipartimento di Chimica e Tecnologie del Farmaco, Dipartimento di Eccellenza 2018-2022, \\ Sapienza Università di Roma, 00185 Rome, Italy; andrea.calcaterra@uniroma1.it (A.C.); \\ giancarlo.fabrizi@uniroma1.it (G.F.); andrea.fochetti@uniroma1.it (A.F.); federico.marrone@uniroma1.it (F.M.); \\ giulia.mazzoccanti@uniroma1.it (G.M.); andrea.serraiocco@uniroma1.it (A.S.) \\ * Correspondence: antonella.goggiamani@uniroma1.it (A.G.); antonia.iazzetti@uniroma1.it (A.I.)
}

check for updates

Citation: Arcadi, A.; Calcaterra, A.; Fabrizi, G.; Fochetti, A.; Goggiamani, A.; Iazzetti, A.; Marrone, F.; Marsicano, V.; Mazzoccanti, G.; Serraiocco, A. Synthesis of

4-Substituted-1,2-Dihydroquinolines by Means of Gold-Catalyzed Intramolecular Hydroarylation Reaction of $\mathrm{N}$-Ethoxycarbonyl- $\mathrm{N}$ Propargylanilines. Molecules 2021, 26, 3366. https://doi.org/10.3390/ molecules26113366

Academic Editor: Michal Szostak

Received: 7 May 2021

Accepted: 1 June 2021

Published: 2 June 2021

Publisher's Note: MDPI stays neutral with regard to jurisdictional claims in published maps and institutional affiliations.

Copyright: (c) 2021 by the authors. Licensee MDPI, Basel, Switzerland. This article is an open access article distributed under the terms and conditions of the Creative Commons Attribution (CC BY) license (https:// creativecommons.org/licenses/by/ $4.0 /)$.

\begin{abstract}
An alternative $\mathrm{Au}(\mathrm{I})$-catalyzed synthetic route to functionalized 1,2-dihydroquinolines is reported. This novel approach is based on the use of $\mathrm{N}$-ethoxycarbonyl protected- $\mathrm{N}$-propargylanilines as building blocks that rapidly undergo the IMHA reaction affording the 6-endo cyclization product in good to high yields. In the presence of $N$-ethoxycarbonyl- $N$-propargyl-meta-substituted anilines, the regiodivergent cyclization at the ortho-/para-position is achieved by the means of catalyst fine tuning.
\end{abstract}

Keywords: gold catalysis; intramolecular hydroarylation; 1,2-dihydroquinolines

\section{Introduction}

4-Substituted-1,2-dihydroquinolines represent key structural units in a variety of naturally occurring products/pharmaceuticals and are used as building blocks in organic synthesis [1-5]. Many methods for the synthesis of functionalized 1,2-dihydroquinolines are known, [1], but due to their pharmaceutical relevance, the development of practical approaches using mild reaction conditions remains an active research area [6-11]. Among them, the transition metal-catalyzed as well as the metal-free mediated intramolecular hydroarylation (IMHA) reactions involving the activation of the $\mathrm{N}$-substituted- $\mathrm{N}$ propargyl anilines carbon-carbon triple bond by using an electrophilic source have been extensively used [12,13]. In particular, the synthetic potential of gold catalysis in the IMHA of $N$-tosyl- $N$-propargylanilines was explored and the corresponding 4-substituted1,2-dihydroquinoline derivatives were efficiently isolated (Scheme 1a) [14-16]. Alternatively these latter products can be obtained by the sequential catalyzed IMHA/Pdcatalyzed cross-coupling of 3-bromo-2-propynyl- $N$-tosylanilines, which afforded the corresponding 4-substituted-1,2-dihydroquinoline derivatives [17,18]. However, the behavior of the substituent attached to $\mathrm{N}$-propargylaniline nitrogen has a significant impact on the reaction outcome. While $\mathrm{N}$-propargylanilines bearing the more easily removable 2nitrobenzenesulfonyl (Ns) nitrogen protecting group underwent the gold-catalyzed IMHA to give the corresponding dihydroquinoline in good yield, subjection of the $\mathrm{N}$-Boc protected derivatives under the same reaction conditions afforded the divergent formation of an oxazolidinone derivative as the exclusive product [16]. Moreover, 1-azaspirotrienone derivatives were produced exclusively instead of the expected dihydroquinolines when $\mathrm{N}$ (4-methoxyphenyl)- $\mathrm{N}$-(3-substituted-2-propyn-1-yl)triflamides were reacted with 2 equiv. of $\mathrm{ICl}$ in $\mathrm{CH}_{2} \mathrm{Cl}_{2}$ at $-78{ }^{\circ} \mathrm{C}$ for 0.5 [19]. As part of our ongoing interest on the development of efficient atom-economical routes of heterocycles by means of gold-catalyzed IMHA [20-22], we envisaged that the introduction of the more suitable ethyl carbamate 
protecting group could allow for some of the drawbacks of the previously reported goldcatalyzed IMHA of $N$-substituted- $N$-propargylanilines (Scheme $1 \mathrm{~b}$ ) to be overcome. The carbamate motif, indeed, in addition to being widely known as excellent protecting groups for amines in organic synthesis, has received a great deal of attention in drug design and medicinal chemistry for its application in the construction of drugs and prodrugs [23].

\section{Previous Works}

a)

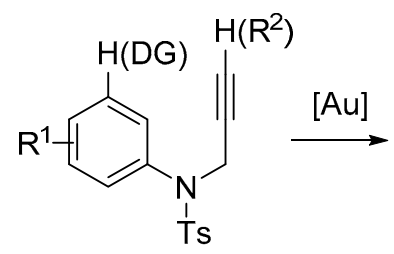<smiles>[R]C1=CCN([13F])c2cc([18OH])[R17]c21</smiles>

and/or $\mathrm{R}^{1}$ t<smiles>[R]C1=CCN([Y5])c2cc[R11]c([OH2+])c21</smiles>

\section{This Work}

b)<smiles>[R]c1cccc(N(CC#C[Al])C(=O)OCC)c1</smiles>

$[\mathrm{Au}]$<smiles>[R]c1[R]cc2c(c1)N(C(=O)OCC)CC=C2[Al]</smiles>

$\mathrm{R}$<smiles></smiles>

Scheme 1. Gold(I)-catalyzed IMHA of N-tosyl- (a) vs. N-ethoxy carbonyl-N-propargylanilines (b).

Herein, we report the results of our investigations.

\section{Results and Discussion}

We started our study by examining the transformation of the $N$-ethoxycarbonyl$\mathrm{N}$-propargylaniline 1a into 2a under different reaction conditions. The results of this preliminary screening are summarized in Table 1.

Table 1. Screening optimal conditions for the IMHA of the $N$-ethoxycarbonyl- $N$-propargylaniline $1 \mathbf{a}^{\text {a }}$.<smiles>CCOC(=O)N(CC#Cc1ccccc1)c1ccccc1</smiles>

$1 \mathrm{a}$

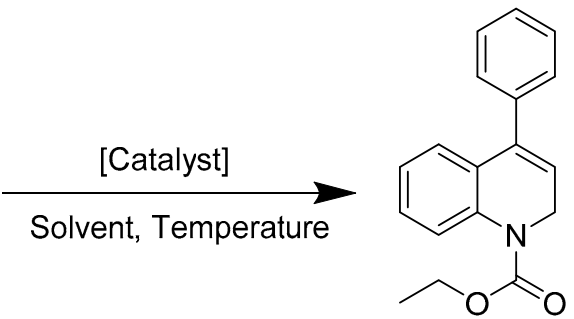

$2 a$

\begin{tabular}{cccccc}
\hline Entry & Catalyst & $\left.\mathbf{T ~}{ }^{\circ} \mathbf{C}\right)$ & Solvent & Time (h) & 2a (Yield \%) \\
\hline 1 & $\mathrm{PtCl}_{2}$ & 80 & $\mathrm{EtOH}$ & 24 & $-\mathrm{b}$ \\
2 & $\mathrm{NaAuCl}_{4}$ & 80 & $\mathrm{EtOH}$ & 48 & $-\mathrm{c}$ \\
3 & $\mathrm{JPAu}\left(\mathrm{CH}_{3} \mathrm{CN}\right) \mathrm{SbF}_{6}$ & 80 & $\mathrm{DCM}^{\mathrm{d}}$ & 1 & 98 \\
4 & $\mathrm{JPAu}^{\mathrm{C}}\left(\mathrm{CH}_{3} \mathrm{CN}\right) \mathrm{SbF}_{6}$ & 100 & $\mathrm{CHCl}_{3}$ & 4.5 & $70^{\mathrm{e}}$ \\
5 & $\mathrm{JPAu}^{\mathrm{f}}\left(\mathrm{CH}_{3} \mathrm{CN}\right) \mathrm{SbF}_{6}$ & 100 & $\mathrm{CHCl}_{3}{ }^{\mathrm{d}}$ & 0.75 & $86^{\mathrm{f}}$ \\
6 & $\mathrm{JPAuCl} / \mathrm{AgNTf}_{2}$ & 80 & $\mathrm{CH}_{2} \mathrm{Cl}_{2}$ & 3.5 & 94 \\
\hline
\end{tabular}

a Reactions were carried out on a $0.35 \mathrm{mmol}$ of $1 \mathrm{a}$ in $2 \mathrm{~mL}$ of solvent in the presence of $0.014 \mathrm{mmol}$ of the catalyst ${ }^{\mathrm{b}} \mathbf{1 a}$ was recovered in 30\% yield, ethyl 3-oxo-3-phenylpropyl(phenyl)carbamate $3 \mathbf{a}$ was isolated in $35 \%$ yield; ${ }^{\mathrm{c}} \mathbf{1 a}$ was recovered in $18 \%$ yield, $3 \mathbf{a}$ was isolated in $75 \%$ yield; ${ }^{\mathrm{d}}$ Commercial anhydrous solvent; ${ }^{\mathrm{e}} \mathbf{3 a}$ a was isolated in $21 \%$ yield; ${ }^{\mathrm{f}}$ 3a was isolated in $7 \%$ yield. 
As shown, the IMHA of 1a occurred in almost quantitative yield in the presence of the commercially available JohnPhosAu( $\mathrm{MeCN}) \mathrm{SbF}_{6}$ catalyst $(4 \mathrm{~mol} \%)$ in anhydrous DCM at $80^{\circ} \mathrm{C}$ (Table 1 , entry 3) $[15,16]$.

About the same result was obtained using the catalytic system JPAuCl/ $\mathrm{AgNTf}_{2}$ (Table 1, entry 6) while slightly poorer results were observed when $\mathrm{CHCl}_{3}$ was used as the solvent instead of DCM (Table 1, entries 4, 5). In this latter solvent, the hydration derivative ethyl 3-oxo-3-phenylpropyl(phenyl)carbamate 3a was isolated to some extent (Figure 1).<smiles>CCOC(=O)N(CCC(=O)c1ccccc1)c1ccccc1</smiles>

3a

Figure 1. Structure of the hydration derivative 3 a.

In contrast with the good efficiency showed by $\mathrm{NaAuCl}_{4} \cdot 2 \mathrm{H}_{2} \mathrm{O}$ in the sequential alkylation/gold-catalyzed annulation reactions of anilines with propargylic bromide derivatives providing quinoline scaffolds in ethanol [24], this gold salt was ineffective as the catalyst of the IMHA of $\mathrm{N}$-ethoxycarbonyl- $\mathrm{N}$-propargylaniline 1a, affording only the formation of the hydration product 3a in good yield (Table 1, entry 2) [25]. Starting material 1a was recovered in almost quantitative yield when $\mathrm{PtCl}_{2}$ was used as the catalyst in ethanol (Table 1, entry 1) [26].

Then, to briefly explore the influence of the protecting group on the reaction outcome, we used the optimized reaction condition for the cyclization of the $\mathrm{N}$-propargylaniline derivatives $4 \mathbf{a}$ and $\mathbf{4 b}$ (Scheme 2).<smiles>[R]N(CC#Cc1ccccc1)c1ccccc1</smiles>

4a: $\mathrm{R}^{3}=\mathrm{COCF}_{3}$ 4b: $\mathrm{R}^{3}=\mathrm{H}$<smiles>CN1CC=C(c2ccccc2)c2ccccc21</smiles>

5a: $\mathrm{R}^{3}=\mathrm{COCF}_{3}(2 \mathrm{~h}$, yield $=0 \%)$

5b: $R^{3}=H(0.5 h$, yield $=25 \%)$

Scheme 2. JPAu $\left(\mathrm{CH}_{3} \mathrm{CN}\right) \mathrm{SbF}_{6}$ catalyzed IMHA of $N$-propargylaniline derivatives $\mathbf{4 a}$ and $\mathbf{4 b}{ }^{\text {a }}$. a Compound $4 \mathrm{~b}$ was recovered in almost quantitative yield when the reaction was carried out in the absence of the catalyst.

As shown by the results reported in Scheme 2, the $N$-trifluoroacetyl- $N$-propargylaniline derivative failed to undergo the desired gold-catalyzed IMHA to give the corresponding dihydroquinoline $5 \mathrm{a}$ in the presence of $4 \mathrm{~mol} \%$ of $\mathrm{JPAu}\left(\mathrm{CH}_{3} \mathrm{CN}\right) \mathrm{SbF}_{6}$ in $\mathrm{DCM}$ at $80{ }^{\circ} \mathrm{C}$. Interestingly, under the same reaction conditions, the simple $N$-(3-phenylprop-2-yn-1-yl)aniline $\mathbf{4 b}$ underwent a complete gold-catalyzed IMHA, but the 4-phenyl-1,2-dihydroquinoline $5 \mathbf{b}$ ( $25 \%$ yield) was prone to be partially oxidized under the reaction conditions to give the corresponding 4-phenylquinoline $\mathbf{6} \mathbf{b}$ ( $56 \%$ yield). The partial oxidation of $5 \mathbf{b}$ to $6 \mathbf{b}$ occurs even under a nitrogen atmosphere. Furthermore, we observed the formation of $\mathbf{7 b}$, which was isolated in 7\% of yield (see Figure 2) [27]. 
<smiles>c1ccc(-c2ccnc3ccccc23)cc1</smiles>

$6 b$<smiles>c1ccc(C2CCNc3ccccc32)cc1</smiles>

$7 b$

Figure 2. Structure of the 4-phenylquinoline $\mathbf{6 b}$ and 4-phenyl-1,2,3,4-tetrahydroquinoline $\mathbf{7 b}$ obtained from compound $4 \mathrm{~b}$ in the condition reported in Scheme 2.

Subsequently, we continued to establish the scope and the generality of gold(I) catalyzed-IMHA reactions of aryl-substituted $N$-ethoxycarbonyl- $N$-propargylanilines $\mathbf{1}$ in terms of ring substitution. The utilization of electron-deficient substrates and the control of the regioselectivity of substituted aromatics remain challenges of gold(I) catalyzed-IMHA reactions of aryl-substituted $\mathrm{N}$-propargylanilines. To that end, a range of readily accessible derivatives $1 \mathbf{a}-\mathbf{j}$ were prepared and then subjected to the IMHA in $\mathrm{CH}_{2} \mathrm{Cl}_{2}$ at $80{ }^{\circ} \mathrm{C}$ in the presence of the JohnPhosAu( $\left.\mathrm{CH}_{3} \mathrm{CN}\right) \mathrm{SbF}_{6}$ as the catalyst. The outcomes of such studies are shown in Table 2. The 4-arylsubstituted-1,2-dihydroquinoline derivatives 2 were isolated in high yields both when the electron donating-OMe group or the strong withdrawing -COOMe were introduced into the para-position of the aromatic ring attached to the alkyne (Table 2, entries 2, 3). Conversely, the introduction of substituents onto the aromatic ring attached to the nitrogen moiety had a different pronounced effect according to their electronic features. The formation of the target 4-aryl-1,2-dihydroquinoline derivative 2 efficiently occurred by the introduction of an electron-donating group on the phenyl ring para to the nitrogen and in the para position of both aromatic rings of the starting arylsubstituted propargylic aniline derivatives (Table 2, entries 4-6). Moreover, the IMHA was also allowed in almost quantitative yield in the presence of the -Me group on the phenyl ring para to the nitrogen and of a withdrawing carbonyl in the para position of the other aryl group (Table 2, entry 7). In absolute agreement with considerations of the positive effect of electronic releasing groups on the aromatic ring attached to the nitrogen on the gold-catalyzed IMHA of substrate $\mathbf{1}$, substrate $\mathbf{1 h}$ bearing two methyl groups on the same benzene nucleus was smoothly converted to the corresponding 1,2-dihydroquilonine $2 \mathbf{h}$ in about quantitative yield either by the gold-catalyzed IMHA (Table 2, entry 8). Substrate 1i, possessing a Cl-substituent on the same aromatic ring, cyclized as expected to afford the corresponding dihydroquinoline derivative $2 \mathbf{i}$ in moderate yield (Table 2, entry 9). The formation of the IMHA products occurred only in low yield in the presence of the strong electron-withdrawing $\mathrm{CF}_{3}$-substituent probably due to the poorer coordination of the alkyne moiety with the gold catalyst (Table 2, entry 10).

With regard to the regiochemical outcome, the meta-substituted derivatives $\mathbf{1 k}-\mathbf{n}$ mainly underwent the para-position cyclization to give the corresponding 1,2-dihydroquinolines $\mathbf{2 k}-\mathbf{n}$ in the presence of JohnPhosAu( $\left.\mathrm{CH}_{3} \mathrm{CN}\right) \mathrm{SbF}_{6}$ (catalyst $\mathbf{A}$ ). Fine tuning factors such as valency state, counterion, and auxiliary ligand in homogeneous gold catalysis is imperative in controlling the product divergence [28]. Indeed, for compounds $\mathbf{1 k}-\mathbf{1}$, the para-position cyclization was revealed to be enhanced in the presence of catalyst $\mathbf{A}^{\prime}$ bearing $\mathrm{NTf}_{2}$ as counterion (catalyst $\mathbf{A}^{\prime}$, entries 2 and 6). The electron-rich tri-isopropylphenyl ring on the ligand and the slightly more strongly coordinated $\mathrm{NTf}_{2}{ }^{-}$jointly lower the electrophilicity of the gold center. On the other hand, the regiodivergent cyclization to the sterically hindered ortho-position to give the regioisomeric 1,2-dihydroquinolines $\mathbf{2}^{\prime} \mathbf{k}-\mathbf{n}$ resulted governed by the electron-deficient ligand features, according to the literature (Table 3) [29]. 
Table 2. Scope of the gold(I)-catalyzed IMHA of the $N$-ethoxycarbonyl- $N$-propargylanilines $\mathbf{1}^{\mathrm{a}}$.<smiles>[R]OC(=O)N(CC#Cc1ccc([R])cc1)c1ccc([R])cc1</smiles>

1

2

\begin{tabular}{cccccc}
\hline Entry & $\mathbf{R}$ & $\mathbf{R}^{\mathbf{2}}$ & $\mathbf{1}$ & Time (h) & $\mathbf{2}$ (Yield \%) \\
\hline 1 & $\mathrm{H}$ & $\mathrm{H}$ & $\mathbf{a}$ & 1 & $\mathbf{2 a}(98)$ \\
2 & $\mathrm{H}$ & $\mathrm{OMe}$ & $\mathbf{b}$ & 18 & $\mathbf{2 b}(82)$ \\
3 & $\mathrm{H}$ & $\mathrm{COMe}$ & $\mathbf{c}$ & 2 & $\mathbf{2 c}(99)$ \\
4 & $4-\mathrm{OMe}$ & $\mathrm{H}$ & $\mathbf{d}$ & 2 & $\mathbf{2 d}(82)$ \\
5 & $4-\mathrm{Me}$ & $\mathrm{H}$ & $\mathbf{e}$ & 1 & $\mathbf{2 e}(99)$ \\
6 & $4-\mathrm{Me}$ & $\mathrm{OMe}$ & $\mathbf{f}$ & 5 & $\mathbf{2 f}(68)^{\mathbf{b}}$ \\
7 & $4-\mathrm{Me}$ & $\mathrm{COMe}$ & $\mathbf{g}$ & 1 & $\mathbf{2 g}(99)$ \\
8 & $3,5-(\mathrm{Me})_{2}$ & $\mathrm{H}$ & $\mathbf{h}$ & 1 & $\mathbf{2 h}(99)$ \\
9 & $4-\mathrm{Cl}$ & $\mathrm{H}$ & $\mathbf{i}$ & 24 & $\mathbf{2 i}(56)^{\mathrm{c}}$ \\
10 & $4-\mathrm{CF}_{3}$ & $\mathrm{OMe}$ & $\mathbf{j}$ & 24 & $\mathbf{2 j}(10)^{\mathrm{d}}$ \\
\hline
\end{tabular}

a Reactions were carried out on $0.35 \mathrm{mmol}$ scale of $\mathbf{1}$ in $2 \mathrm{~mL}$ of $\mathrm{CH}_{2} \mathrm{Cl}_{2}$; ${ }^{\mathrm{b}}$ The starting alkyne $\mathbf{1 f}$ was recovered in $8 \%$ yield; ${ }^{\mathrm{c}}$ The starting alkyne $1 \mathbf{i}$ was recovered in $18 \%$ yield; ${ }^{\mathrm{d}}$ The starting alkyne $\mathbf{1} \mathbf{j}$ was recovered in $60 \%$ yield.

Very likely, the control of ortho/para site-selectivity in these substrates is the result of the different coordination modes of the gold catalyst influenced by sterics and electronics of the auxiliary ligand. The prowess of electron-rich bulk ligands in pushing the $\pi$-system toward the para $\mathrm{C}-\mathrm{H}$ bond through a $\mathrm{Au}(\mathrm{I})$-bicoordinate activation was also explored in the 6-endo-dig gold catalyzed hydroarylation of functionalized $N$-aryl alkynamides (Figure 3) [30].<smiles></smiles><smiles>[R]c1ccc(C#CCN(C(=O)OCC)c2c([2H])ccc([R1])c2[14CH3])cc1</smiles>

Figure 3. Ligand control of ortho/para site-selectivity.

Indeed, according to the literature [13], the gold catalyzed IMHA proceeds through a Friedel-Crafts type mechanism: $\eta^{2}$-coordination of alkyne moiety affords complex I, which undergoes an electrophilic aromatic substitution to give the Wheland-type intermediate II. This latter, after aromatization and protodeauration would give the product 2 . The proposed mechanism is outlined in the Scheme 3. 
Table 3. Ortho- vs. para-position annulation in the gold(I)-catalyzed IMHA of the $N$-ethoxycarbonyl$N$-propargylanilines $\mathbf{1} \mathbf{k}-\mathbf{n}^{\text {a }}$.<smiles>[R]CON(CC#Cc1ccc([R])cc1)c1cccc([R])c1</smiles>

$1 k-n$<smiles>CC(C)(C)[GeH3]</smiles><smiles>[R]c1ccc(C2=CCN(COCC)c3cc([R])ccc32)cc1</smiles>

2k-n<smiles>[R]c1ccc(C2=CCN(CC)c3cccc([R])c32)cc1</smiles>

2'k-n

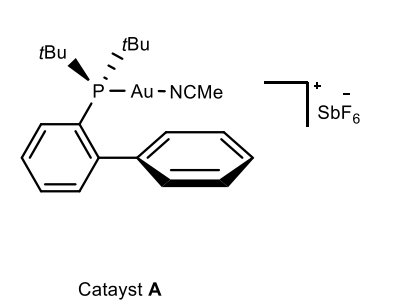

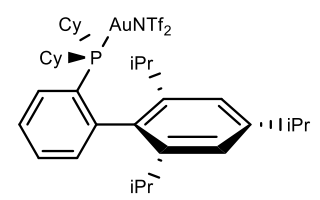

Catayst $\mathbf{A}^{\prime}$

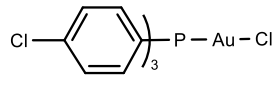

AgSbF6

Catalyst B

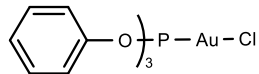

AgSbF6

\begin{tabular}{|c|c|c|c|c|c|c|}
\hline Entry & $\mathbf{R}^{1}$ & $\mathbf{R}^{2}$ & Catalyst & Overall Yield (\%) & $2 / 2^{\prime}$ & $\overline{\text { (Ratio) }^{c}}$ \\
\hline 1 & \multirow{4}{*}{$\mathrm{OMe}$} & \multirow{4}{*}{$\mathrm{H}$} & A & 99 & \multirow{4}{*}{$2 \mathrm{k} / 2^{\prime} \mathrm{k}$} & $(67 / 33)$ \\
\hline 2 & & & $\mathrm{~A}^{\prime}$ & 99 & & $(94 / 6)$ \\
\hline 3 & & & B & 90 & & $(44 / 56)$ \\
\hline 4 & & & C & 67 & & $(46 / 54)$ \\
\hline 5 & \multirow{4}{*}{$\mathrm{OMe}$} & \multirow{4}{*}{ COMe } & A & 99 & \multirow{4}{*}{$21 / 2^{\prime} 1$} & $(61 / 39)$ \\
\hline 6 & & & $\mathrm{~A}^{\prime}$ & 99 & & $(75 / 25)$ \\
\hline 7 & & & B & 99 & & $(54 / 46)$ \\
\hline 8 & & & C & 86 & & $(54 / 46)$ \\
\hline 9 & \multirow{4}{*}{$\mathrm{OMe}$} & \multirow{4}{*}{$\mathrm{OMe}$} & A & 70 & \multirow{4}{*}{$2 \mathrm{~m} / 2^{\prime} \mathrm{m}$} & $(91 / 9)$ \\
\hline 10 & & & $A^{\prime}$ & 73 & & $(91 / 9)$ \\
\hline 11 & & & B & 83 & & $(63 / 37)$ \\
\hline 12 & & & C & 77 & & $(51 / 49)$ \\
\hline 13 & \multirow{4}{*}{$\mathrm{COMe}$} & \multirow{4}{*}{ COOMe } & A & 85 & \multirow{4}{*}{$2 n / 2^{\prime} n$} & $(88 / 12)$ \\
\hline 14 & & & $\mathrm{~A}^{\prime}$ & 75 & & $(64 / 36)$ \\
\hline 15 & & & B & 99 & & $(40 / 60)$ \\
\hline 16 & & & C & 72 & & $(19 / 81)$ \\
\hline 17 & \multirow{4}{*}{$\mathrm{COMe}$} & \multirow{4}{*}{ COMe } & A & 88 & \multirow{4}{*}{$20 / 2^{\prime} \mathrm{o}$} & $(88 / 12)$ \\
\hline 18 & & & $A^{\prime}$ & 90 & & $(65 / 35)$ \\
\hline 19 & & & B & 70 & & $(33 / 67)$ \\
\hline 20 & & & C & 82 & & $(20 / 80)$ \\
\hline
\end{tabular}

${ }^{a}$ Reactions were carried out on $0.35 \mathrm{mmol}$ of $\mathbf{1 k}-\mathbf{n}$ in $2 \mathrm{~mL}$ in $\mathrm{CH}_{2} \mathrm{Cl}_{2} ;{ }^{\mathrm{b}}$ Overall yield refers to the mixture of regioisomers $2+2^{\prime} ;{ }^{c}$ The isomeric ratio was determined by ${ }^{1} \mathrm{H}$ NMR analyses. 


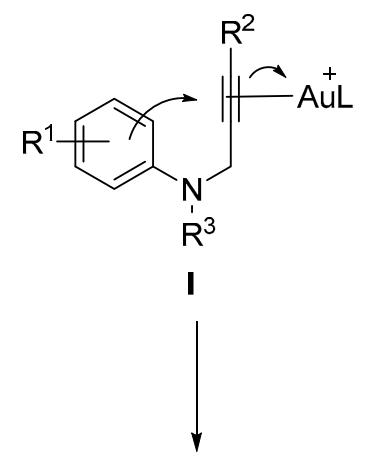

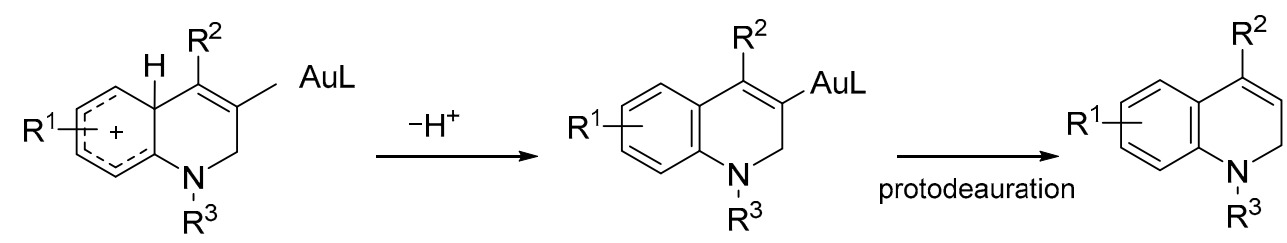

Wheland-type intermediate

II

III

Scheme 3. Proposed Friedel-Crafts mechanism for the Au(I)-catalyzed cyclization of N-substituted$N$-propargylanilines.

\section{Materials and Methods}

\subsection{General Information}

All the commercially available reagents, catalysts, bases, and solvents were used as purchased without further purification. Reaction products $2 \mathbf{a}-\mathbf{e}$ and $\mathbf{2} \mathbf{g}-\mathbf{h}$ were filtered on a pad of $\mathrm{SiO}_{2}$ using AcOEt, while reaction products $2 \mathbf{f}, \mathbf{2} \mathbf{i}$ and $\mathbf{2} \mathbf{j}$ were purified by chromatography on $\mathrm{SiO}_{2}(25-40 \mu \mathrm{m})$, eluting with $n$-hexane/AcOEt mixtures. Reaction products $2 \mathbf{k} / \mathbf{2}^{\prime} \mathbf{k}-\mathbf{2 o} / \mathbf{2}^{\prime} \mathbf{o}$ were obtained as isomeric mixtures by filtration on a pad of $\mathrm{SiO}_{2}$ using AcOEt to eliminate the catalysts before calculating the isomeric ratio by ${ }^{1} \mathrm{H} N M R$. When possible, to obtain suitable NMR spectra of each compound, the isomeric mixtures were further purified by semi-preparative HPLC under normal phase condition using a Nucleodur $100-5$ column $(762,007.100)$ and eluting with $n$-hexane/AcOEt mixtures. ${ }^{1} \mathrm{H}$ NMR $(400.13 \mathrm{MHz}),{ }^{13} \mathrm{C}$ NMR $(100.6 \mathrm{MHz})$, and ${ }^{19} \mathrm{~F}$ spectra $(376.5 \mathrm{MHz})$ were recorded with a Bruker Avance 400 spectrometer. Splitting patterns were designed as s (singlet), $\mathrm{d}$ (doublet), $\mathrm{t}$ (triplet), $\mathrm{q}$ (quartet), $\mathrm{m}$ (multiplet), or bs (broad singlet). IR spectra were recorded with a Jasco FT/IR-430 spectrometer. HRMS were recorded with an Orbitrap Exactive Mass spectrometer with ESI source. Melting points were determined with a Büchi B-545 apparatus and are uncorrected.

\subsection{Synthetic Procedures}

\subsubsection{Preparation of Substrates 1}

Substrates were prepared as described in the Supplementary Materials.

3.2.2. Preparation of Derivatives 2: Typical Procedure for the Preparation of the Ethyl 4-Phenylquinoline-1(2H)-Carboxylate 2a

A Carousel Tube Reactor (Radley Discovery Technology) equipped with a magnetic stirring bar was charged with ethyl phenyl(3-phenylprop-2-yn-1-yl)carbamate $1 \mathbf{a}(97.8 \mathrm{mg}$, $0.35 \mathrm{mmol}, 1$ equiv.), $\mathrm{CH}_{2} \mathrm{Cl}_{2}(2 \mathrm{~mL})$, JohnPhosAu(MeCN)SbF 6 (10.8 mg, $0.014 \mathrm{mmol}$, 0.04 equiv.), and sealed. Then, the reaction mixture was stirred at $80^{\circ} \mathrm{C}$ and monitored by TLC until the disappearance of the starting material. After $1 \mathrm{~h}$, the obtained mixture was cooled at room temperature and concentrated under reduced pressure. The residue was filtered on a pad of $\mathrm{SiO}_{2}$ to afford $97.1 \mathrm{mg}$ of ethyl 4-phenylquinoline-1(2H)-carboxylate $\mathbf{2 a}$ (99\% yield). 
Compound 2a: yield: 99\% (97.1 mg); yellow oil; IR (neat): 2912, 1707, $1380 \mathrm{~cm}^{-1}$; ${ }^{1} \mathrm{H} \mathrm{NMR}(400.13 \mathrm{MHz})\left(\mathrm{CDCl}_{3}\right): \delta=7.69(\mathrm{~d}, J=7.4 \mathrm{~Hz}, 1 \mathrm{H}), 7.45-7.36(\mathrm{~m}, 5 \mathrm{H}), 7.31-7.27(\mathrm{~m}$, $1 \mathrm{H}), 7.12-7.05(\mathrm{~m}, 2 \mathrm{H}), 6.07(\mathrm{t}, J=4.5 \mathrm{~Hz}, 1 \mathrm{H}), 4.51(\mathrm{~d}, J=4.5 \mathrm{~Hz}, 2 \mathrm{H}), 4.33(\mathrm{q}, J=7.1 \mathrm{~Hz}$, 2H), $1.39(\mathrm{t}, J=7.1 \mathrm{~Hz}, 3 \mathrm{H}) ;{ }^{13} \mathrm{C}$ NMR $(100.6 \mathrm{MHz})\left(\mathrm{CDCl}_{3}\right): \delta=154.2(\mathrm{q}), 138.9(\mathrm{q}), 137.3$ (q), $129.3(\mathrm{q}), 128.8(\mathrm{CH}), 128.5(\mathrm{CH}), 127.9(\mathrm{CH}), 127.7(\mathrm{CH}), 126.1(\mathrm{CH}), 124.2(\mathrm{CH}), 124.1$ $(\mathrm{CH}), 123.4(\mathrm{CH}), 62.3\left(\mathrm{CH}_{2}\right), 43.1\left(\mathrm{CH}_{2}\right), 14.7\left(\mathrm{CH}_{3}\right) ; \mathrm{MS}$ (EI ion source): $m / z(\%)=279$ (18, [M+ $]$ ), 206 (100), 204 (62), 102 (21); HRMS: $m / z$ [M + Na] ${ }^{+}$calcd. for $\mathrm{C}_{18} \mathrm{H}_{17} \mathrm{NO}_{2} \mathrm{Na}$ : 302.1152; found: 302.1152 .

\subsection{Characterization Data}

\subsubsection{Characterization Data of Compound 1a-o}

Characterization data of substrate $\mathbf{1}$ are reported in the Supplementary Materials.

\subsubsection{Characterization Data of Compound $\mathbf{2 b}-\mathbf{j}, \mathbf{2} \mathbf{2}-\mathbf{0}, \mathbf{2}^{\prime} \mathbf{1}-\mathbf{2}^{\prime} \mathbf{o}$}

Compound 2b. Yield: $82 \%$ (88.9 mg); yellow oil; IR (neat): 2980, 1699, 1608, 1510, $1051 \mathrm{~cm}^{-1} ;{ }^{1} \mathrm{H}$ NMR $(400.13 \mathrm{MHz})\left(\mathrm{CDCl}_{3}\right): \delta=7.56(\mathrm{bd}, J=7.7 \mathrm{~Hz}, 1 \mathrm{H}), 7.19(\mathrm{~d}, J=8.8 \mathrm{~Hz}$, $2 \mathrm{H}), 7.18-7.15(\mathrm{~m}, 1 \mathrm{H}), 7.01\left(\mathrm{dd}, J_{1}=7.8 \mathrm{~Hz}, J_{2}=1.7 \mathrm{~Hz}, 1 \mathrm{H}\right), 6.95\left(\mathrm{dt}, J_{1}=7.2 \mathrm{~Hz}, J_{2}=1.2 \mathrm{~Hz}\right.$, $1 \mathrm{H}), 6.84(\mathrm{~d}, J=8.8 \mathrm{~Hz}, 2 \mathrm{H}), 5.91(\mathrm{t}, J=4.5 \mathrm{~Hz}, 1 \mathrm{H}), 4.37(\mathrm{~d}, J=4.5 \mathrm{~Hz}, 2 \mathrm{H}), 4.20(\mathrm{q}, J=7.1 \mathrm{~Hz}$, 2H), $3.76(\mathrm{~s}, 3 \mathrm{H}), 1.26(\mathrm{t}, J=7.1 \mathrm{~Hz}, 3 \mathrm{H}) ;{ }^{13} \mathrm{C}$ NMR $(100.6 \mathrm{MHz})\left(\mathrm{CDCl}_{3}\right): \delta=159.4(\mathrm{q}), 154.2$ $(\mathrm{q}), 138.4(\mathrm{q}), 137.4(\mathrm{q}), 131.3(\mathrm{q}), 130.0(\mathrm{CH}), 129.5(\mathrm{q}), 127.6(\mathrm{CH}), 126.1(\mathrm{CH}), 124.15(\mathrm{CH})$, $124.08(\mathrm{CH}), 122.5(\mathrm{CH}), 113.9(\mathrm{CH}), 62.2\left(\mathrm{CH}_{2}\right), 55.4\left(\mathrm{CH}_{3}\right), 43.1\left(\mathrm{CH}_{2}\right), 14.7\left(\mathrm{CH}_{3}\right)$; MS (EI ion source): $m / z(\%)=309\left(47,\left[\mathrm{M}^{+}\right]\right), 280(93), 236(100), 221$ (21), 192 (20); HRMS: $m / z$ $[\mathrm{M}+\mathrm{Na}]^{+}$calcd. for $\mathrm{C}_{19} \mathrm{H}_{19} \mathrm{NO}_{3} \mathrm{Na}$ : 332.1257; found: 332.1261 .

Compound 2c. Yield: 99\% (111.3 mg); white solid; m.p. = 110-111 ${ }^{\circ} \mathrm{C}$; IR (neat): 2977, $1698,1604,1484 \mathrm{~cm}^{-1} ;{ }^{1} \mathrm{H}$ NMR $(400.13 \mathrm{MHz})\left(\mathrm{CDCl}_{3}\right): \delta=7.98(\mathrm{~d}, J=8.5 \mathrm{~Hz}, 2 \mathrm{H}), 7.67-7.65$ $(\mathrm{m}, 1 \mathrm{H}), 7.45(\mathrm{~d}, J=8.5 \mathrm{~Hz}, 2 \mathrm{H}), 7.30-7.25(\mathrm{~m}, 1 \mathrm{H}), 7.06-6.99(\mathrm{~m}, 2 \mathrm{H}), 6.10(\mathrm{t}, J=4.6 \mathrm{~Hz}, 1 \mathrm{H})$, $4.49(\mathrm{~d}, J=4.6 \mathrm{~Hz}, 2 \mathrm{H}), 4.28(\mathrm{q}, J=7.1 \mathrm{~Hz}, 2 \mathrm{H}), 2.63(\mathrm{~s}, 3 \mathrm{H}), 1.34(\mathrm{t}, J=7.1 \mathrm{~Hz}, 3 \mathrm{H}) ;{ }^{13} \mathrm{C} \mathrm{NMR}$ $(100.6 \mathrm{MHz})\left(\mathrm{CDCl}_{3}\right): \delta=197.8(\mathrm{q}), 154.2(\mathrm{q}), 143.9(\mathrm{q}), 138.3(\mathrm{q}), 137.4(\mathrm{q}), 136.6(\mathrm{q}), 129.1$ $(\mathrm{CH}), 128.7(\mathrm{CH}), 128.1(\mathrm{CH}), 126.0(\mathrm{CH}), 124.6(\mathrm{CH}), 124.4(\mathrm{CH}), 120.6(\mathrm{q}), 62.4\left(\mathrm{CH}_{2}\right), 43.1$ $\left(\mathrm{CH}_{2}\right), 26.8\left(\mathrm{CH}_{3}\right), 14.7\left(\mathrm{CH}_{3}\right)$; MS (EI ion source): $\mathrm{m} / z(\%)=321\left(27,\left[\mathrm{M}^{+}\right]\right), 292(71), 248$ (100), 205 (28); HRMS: $m / z$ [M + H] $]^{+}$calcd. for $\mathrm{C}_{20} \mathrm{H}_{20} \mathrm{NO}_{3}$ : 322.1438; found: 322.1431.

Compound 2d. Yield: $82 \%$ (88.6 mg); pale yellow oil; IR (neat): 2980, 1702, 1491, $1382 \mathrm{~cm}^{-1} ;{ }^{1} \mathrm{H}$ NMR $(400.13 \mathrm{MHz})\left(\mathrm{CDCl}_{3}\right): \delta=7.56-7.53(\mathrm{~m}, 1 \mathrm{H}), 7.40-7.33(\mathrm{~m}, 5 \mathrm{H}), 6.81$ $\left(\mathrm{dd}, J_{1}=8.9 \mathrm{~Hz}, J_{2}=2.9 \mathrm{~Hz}, 1 \mathrm{H}\right), 6.60(\mathrm{~d}, J=2.9 \mathrm{~Hz}, 1 \mathrm{H}), 6.06(\mathrm{t}, J=4.5 \mathrm{~Hz}, 1 \mathrm{H}), 4.44(\mathrm{~d}$, $J=4.5 \mathrm{~Hz}, 2 \mathrm{H}), 4.26(\mathrm{q}, J=7.1 \mathrm{~Hz}, 2 \mathrm{H}), 3.69(\mathrm{~s}, 3 \mathrm{H}), 1.33(\mathrm{t}, J=7.1 \mathrm{~Hz}, 3 \mathrm{H}) ;{ }^{13} \mathrm{C} \mathrm{NMR}$ $(100.6 \mathrm{MHz})\left(\mathrm{CDCl}_{3}\right): \delta=156.2(\mathrm{q}), 154.4(\mathrm{q}), 138.9(\mathrm{q}), 138.8(\mathrm{q}), 130.51(\mathrm{q}), 130.47(\mathrm{q})$, $128.8(\mathrm{CH}), 128.5(\mathrm{CH}), 127.9(\mathrm{CH}), 125.2(\mathrm{CH}), 113.0(\mathrm{CH}), 111.6(\mathrm{CH}), 62.1\left(\mathrm{CH}_{2}\right), 55.6$ $\left(\mathrm{CH}_{3}\right), 43.2\left(\mathrm{CH}_{2}\right), 14.7\left(\mathrm{CH}_{3}\right)$; MS (EI ion source): $\mathrm{m} / z(\%)=309\left(21,\left[\mathrm{M}^{+}\right]\right), 280(24), 236$ (100), 193 (36), 165 (19), 63 (30); HRMS: $m / z[\mathrm{M}+\mathrm{H}]^{+}$calcd. for $\mathrm{C}_{19} \mathrm{H}_{20} \mathrm{NO}_{3}$ : 310.1438; found: 310.1443 .

Compound 2e. Yield: 99\% (101.4 mg); orange oil; IR (neat): 2981, 1697, 1493, $1378 \mathrm{~cm}^{-1}$; ${ }^{1} \mathrm{H}$ NMR $(400.13 \mathrm{MHz})\left(\mathrm{CDCl}_{3}\right): \delta=7.53-7.52(\mathrm{~m}, 1 \mathrm{H}), 7.42-7.34(\mathrm{~m}, 5 \mathrm{H}), 7.07(\mathrm{dd}$, $\left.J_{1}=8.3 \mathrm{~Hz}, J_{2}=1.4 \mathrm{~Hz}, 1 \mathrm{H}\right), 6.86(\mathrm{bd}, J=1.4 \mathrm{~Hz}, 1 \mathrm{H}), 6.02(\mathrm{t}, J=4.5 \mathrm{~Hz}, 1 \mathrm{H}), 4.45(\mathrm{~d}$, $J=4.5 \mathrm{~Hz}, 2 \mathrm{H}), 4.28(\mathrm{q}, J=7.1 \mathrm{~Hz}, 2 \mathrm{H}), 2.24(\mathrm{~s}, 3 \mathrm{H}), 1.34(\mathrm{t}, J=7.1 \mathrm{~Hz}, 3 \mathrm{H}) ;{ }^{13} \mathrm{C} \mathrm{NMR}$ $(100.6 \mathrm{MHz})\left(\mathrm{CDCl}_{3}\right): \delta=154.2(\mathrm{q}), 139.1(\mathrm{q}), 139.0(\mathrm{q}), 134.8(\mathrm{q}), 133.8(\mathrm{q}), 129.1(\mathrm{q}), 128.8$ $(\mathrm{CH}), 128.5(\mathrm{CH}), 128.4(\mathrm{CH}), 127.8(\mathrm{CH}), 126.5(\mathrm{CH}), 124.0(\mathrm{CH}), 123.5(\mathrm{CH}), 62.2\left(\mathrm{CH}_{2}\right)$, $43.1\left(\mathrm{CH}_{2}\right), 21.1\left(\mathrm{CH}_{3}\right), 14.7\left(\mathrm{CH}_{3}\right)$; MS (EI ion source): $m / z(\%)=293\left(39,\left[\mathrm{M}^{+}\right]\right), 264$ (49), 220 (100), 204 (55), 63 (29); HRMS: $m / z[\mathrm{M}+\mathrm{H}]^{+}$calcd. for $\mathrm{C}_{19} \mathrm{H}_{20} \mathrm{NO}_{2}: 294.1489$; found: 294.1491.

Compound 2f. Yield: 68\% (77.2 mg); yellow oil; IR (neat): 2980, 2836, 1702, 1608, 1509, $1463 \mathrm{~cm}^{-1} ;{ }^{1} \mathrm{H}$ NMR $(400.13 \mathrm{MHz})\left(\mathrm{CDCl}_{3}\right): \delta=7.53-7.51(\mathrm{~m}, 1 \mathrm{H}), 7.28(\mathrm{~d}, J=8.8 \mathrm{~Hz}, 2 \mathrm{H})$, $7.07\left(\mathrm{dd}, J_{1}=8.3 \mathrm{~Hz}, J_{2}=1.6 \mathrm{~Hz}, 1 \mathrm{H}\right), 6.93(\mathrm{~d}, J=8.8 \mathrm{~Hz}, 2 \mathrm{H}), 6.89(\mathrm{bd}, J=1.6 \mathrm{~Hz}, 1 \mathrm{H}), 5.97$ $(\mathrm{t}, J=4.5 \mathrm{~Hz}, 1 \mathrm{H}), 4.43(\mathrm{~d}, J=4.5 \mathrm{~Hz}, 2 \mathrm{H}), 4.27(\mathrm{q}, J=7.1 \mathrm{~Hz}, 2 \mathrm{H}), 3.85(\mathrm{~s}, 3 \mathrm{H}), 2.24(\mathrm{~s}, 3 \mathrm{H})$, $1.34(\mathrm{t}, J=7.1 \mathrm{~Hz}, 3 \mathrm{H}) ;{ }^{13} \mathrm{C}$ NMR $(100.6 \mathrm{MHz})\left(\mathrm{CDCl}_{3}\right): \delta=159.3(\mathrm{q}), 154.2(\mathrm{q}), 138.5(\mathrm{q})$, 
134.8 (q), 133.7 (q), 131.4 (q), $129.9(\mathrm{CH}), 129.3(\mathrm{CH}), 128.3(\mathrm{CH}), 126.5(\mathrm{CH}), 123.9(\mathrm{CH})$, $122.7(\mathrm{q}), 113.9(\mathrm{CH}), 62.1\left(\mathrm{CH}_{2}\right), 55.4\left(\mathrm{CH}_{3}\right), 43.1\left(\mathrm{CH}_{2}\right), 21.1\left(\mathrm{CH}_{3}\right), 14.7\left(\mathrm{CH}_{3}\right)$; MS (EI ion source): $m / z(\%)=323\left(43,\left[\mathrm{M}^{+}\right]\right), 294(87), 250(100), 235$ (20), 207 (17); HRMS: $m / z$ $[\mathrm{M}+\mathrm{H}]^{+}$calcd. for $\mathrm{C}_{20} \mathrm{H}_{22} \mathrm{NO}_{3}$ : 324.1594; found: 324.1597 .

Compound 2g. Yield: 96\% (112.4 mg); orange wax; IR (neat): 2980, 2243, 1705, 1596, $1494 \mathrm{~cm}^{-1} ;{ }^{1} \mathrm{H}$ NMR $(400.13 \mathrm{MHz})\left(\mathrm{CDCl}_{3}\right): \delta=7.99(\mathrm{~d}, J=8.4 \mathrm{~Hz}, 2 \mathrm{H}), 7.54-7.52(\mathrm{~m}, 1 \mathrm{H})$, $7.45(\mathrm{~d}, J=8.4 \mathrm{~Hz}, 2 \mathrm{H}), 7.11-7.07(\mathrm{~m}, 1 \mathrm{H}), 6.79(\mathrm{bd}, J=1.4 \mathrm{~Hz}, 1 \mathrm{H}), 6.08(\mathrm{t}, J=4.4 \mathrm{~Hz}, 1 \mathrm{H})$, $4.46(\mathrm{~d}, J=4.4 \mathrm{~Hz}, 2 \mathrm{H}), 4.27(\mathrm{q}, J=7.1 \mathrm{~Hz}, 2 \mathrm{H}), 2.64(\mathrm{~s}, 3 \mathrm{H}), 2.23(\mathrm{~s}, 3 \mathrm{H}), 1.34(\mathrm{t}, J=7.1 \mathrm{~Hz}$, $3 \mathrm{H}) ;{ }^{13} \mathrm{C}$ NMR (100.6 MHz) $\left(\mathrm{CDCl}_{3}\right): \delta=197.9(\mathrm{q}), 154.2(\mathrm{q}), 144.0(\mathrm{q}), 138.3$ (q), 136.5 (q), 134.8 (q), 133.9 (q), $129.6(\mathrm{CH}), 129.0(\mathrm{CH}), 128.7(\mathrm{CH}), 128.6(\mathrm{CH}), 128.5(\mathrm{q}), 126.3(\mathrm{CH})$, $124.1(\mathrm{CH}), 62.3\left(\mathrm{CH}_{2}\right), 43.1\left(\mathrm{CH}_{2}\right), 26.8\left(\mathrm{CH}_{3}\right), 21.1\left(\mathrm{CH}_{3}\right), 14.7\left(\mathrm{CH}_{3}\right)$; $\mathrm{MS}$ (EI ion source): $m / z(\%)=351\left(\mathrm{M}^{+}, 31\right), 322(50), 278(14), 157(46), 134(100), 114(62) ; \mathrm{HRMS}: m / z[\mathrm{M}+\mathrm{H}]^{+}$ calcd. for $\mathrm{C}_{21} \mathrm{H}_{22} \mathrm{NO}_{3}$ : 336.1594; found: 336.1598 .

Compound 2h. Yield: 99\% (106.3 mg); yellow oil; IR (neat): 2979, 1703, 1608, 1557, 1376, $1271 \mathrm{~cm}^{-1} ;{ }^{1} \mathrm{H}$ NMR $(400.13 \mathrm{MHz})\left(\mathrm{CDCl}_{3}\right): \delta=7.28-7.14(\mathrm{~m}, 6 \mathrm{H}), 6.68(\mathrm{~s}, 1 \mathrm{H}), 6.01$ $(\mathrm{t}, J=5.2 \mathrm{~Hz}, 1 \mathrm{H}), 4.20-4.14(\mathrm{~m}, 4 \mathrm{H}), 2.26(\mathrm{~s}, 3 \mathrm{H}), 1.65(\mathrm{~s}, 3 \mathrm{H}), 1.24(\mathrm{t}, J=7.1 \mathrm{~Hz}, 3 \mathrm{H})$; ${ }^{13} \mathrm{C}$ NMR $(100.6 \mathrm{MHz})\left(\mathrm{CDCl}_{3}\right): \delta=153.9(\mathrm{q}), 141.8(\mathrm{q}), 139.9(\mathrm{q}), 139.1(\mathrm{q}), 137.3(\mathrm{q}), 135.3$ (q), $129.0(\mathrm{CH}), 128.5(\mathrm{CH}), 127.4(\mathrm{CH}), 127.2(\mathrm{CH}), 125.8(\mathrm{q}), 125.3(\mathrm{CH}), 122.4(\mathrm{CH}), 62.1$ $\left(\mathrm{CH}_{2}\right), 42.6\left(\mathrm{CH}_{2}\right), 23.0\left(\mathrm{CH}_{3}\right), 21.5\left(\mathrm{CH}_{3}\right), 14.7\left(\mathrm{CH}_{3}\right)$; $\mathrm{MS}$ (EI ion source): $m / z(\%)=307(20$, $\left.\left[\mathrm{M}^{+}\right]\right), 278$ (27), 234 (100), 218 (18); HRMS: $m / z[\mathrm{M}+\mathrm{H}]^{+}$calcd. for $\mathrm{C}_{20} \mathrm{H}_{22} \mathrm{NO}_{2}$ : 308.1645; found: 308.1649 .

Compound 2i. Yield: 56\% (61.7 mg); pale yellow oil; IR (neat): 2981, 2847, 1702, 1594, $1481 \mathrm{~cm}^{-1} ;{ }^{1} \mathrm{H}$ NMR $(400.13 \mathrm{MHz})\left(\mathrm{CDCl}_{3}\right): \delta=7.59(\mathrm{bd}, J=8.1 \mathrm{~Hz}, 1 \mathrm{H}), 7.43-7.36(\mathrm{~m}$, $3 \mathrm{H}), 7.33-7.30(\mathrm{~m}, 2 \mathrm{H}), 7.21\left(\mathrm{dd}, J_{1}=8.7 \mathrm{~Hz}, J_{2}=2.5 \mathrm{~Hz}, 1 \mathrm{H}\right), 7.02(\mathrm{~d}, J=2.5 \mathrm{~Hz}, 1 \mathrm{H}), 6.06$ $(\mathrm{t}, J=4.5 \mathrm{~Hz}, 1 \mathrm{H}), 4.47(\mathrm{~d}, J=4.5 \mathrm{~Hz}, 2 \mathrm{H}), 4.28(\mathrm{q}, J=7.1 \mathrm{~Hz}, 2 \mathrm{H}), 1.34(\mathrm{t}, J=7.1 \mathrm{~Hz}, 3 \mathrm{H})$; ${ }^{13} \mathrm{C} \mathrm{NMR}(100.6 \mathrm{MHz})\left(\mathrm{CDCl}_{3}\right): \delta=153.9(\mathrm{q}), 138.1(\mathrm{q}), 135.6(\mathrm{q}), 130.7(\mathrm{q}), 129.5(\mathrm{CH}), 128.61$ $(\mathrm{CH}), 128.60(\mathrm{CH}), 128.0(\mathrm{CH}), 127.4(\mathrm{CH}), 125.7(\mathrm{CH}), 125.3(\mathrm{CH}), 124.4(\mathrm{q}), 62.3\left(\mathrm{CH}_{2}\right)$, $43.0\left(\mathrm{CH}_{2}\right), 14.5\left(\mathrm{CH}_{3}\right)$. MS (EI ion source): $m / z(\%)=313\left(30,\left[\mathrm{M}^{+}\right]\right), 284(72), 240(100), 204$ (62), 176 (19); HRMS: $m / z[\mathrm{M}+\mathrm{H}]^{+}$calcd. for $\mathrm{C}_{18} \mathrm{H}_{17} \mathrm{ClNO}_{2}$ : 314.0942; found: 314.0952.

Compound 2j. Yield: 10\% (13.0 mg); colorless oil; IR (eat): 2919, 2848, 1710, 1609, 1382, $1051 \mathrm{~cm}^{-1} ;{ }^{1} \mathrm{H} \mathrm{NMR}(400.13 \mathrm{MHz})\left(\mathrm{CDCl}_{3}\right): \delta=7.77(\mathrm{bd}, J=8.5 \mathrm{~Hz}, 1 \mathrm{H}), 7.49(\mathrm{dd}$, $\left.J_{1}=8.6 \mathrm{~Hz}, J_{2}=1.6 \mathrm{~Hz}, 1 \mathrm{H}\right), 7.33(\mathrm{bd}, J=1.6 \mathrm{~Hz}, 1 \mathrm{H}), 7.25(\mathrm{~d}, J=8.8 \mathrm{~Hz}, 2 \mathrm{H}), 6.94(\mathrm{~d}$, $J=8.8 \mathrm{~Hz}, 2 \mathrm{H}), 6.04(\mathrm{t}, J=4.5 \mathrm{~Hz}, 1 \mathrm{H}), 4.48(\mathrm{~d}, J=4.5 \mathrm{~Hz}, 2 \mathrm{H}), 4.30(\mathrm{q}, J=7.1 \mathrm{~Hz}, 2 \mathrm{H})$, $3.86(\mathrm{~s}, 3 \mathrm{H}), 1.36(\mathrm{t}, J=7.1 \mathrm{~Hz}, 3 \mathrm{H}) ;{ }^{13} \mathrm{C} \mathrm{NMR}(100.6 \mathrm{MHz})\left(\mathrm{CDCl}_{3}\right): \delta=159.7$ (q), 153.9 (q), $140.4(\mathrm{q}), 137.8$ (q), $130.4(\mathrm{q}), 129.9(\mathrm{q}), 129.7(\mathrm{CH}), 126.1(\mathrm{q}, \mathrm{q}, J=32.5 \mathrm{~Hz}), 124.5(\mathrm{CH}, \mathrm{q}$, $J=3.7 \mathrm{~Hz}), 124.20(\mathrm{q}, \mathrm{q}, J=270.3 \mathrm{~Hz}), 124.19(\mathrm{CH}), 123.7(\mathrm{CH}), 123.1(\mathrm{CH}, \mathrm{q}, J=3.7 \mathrm{~Hz})$, $114.2(\mathrm{CH}), 62.7\left(\mathrm{CH}_{2}\right), 55.5\left(\mathrm{CH}_{3}\right), 43.2\left(\mathrm{CH}_{2}\right), 14.6\left(\mathrm{CH}_{3}\right) ;{ }^{19} \mathrm{~F}$ NMR $(376.5 \mathrm{MHz})\left(\mathrm{CDCl}_{3}\right)$ : $\delta=-62.3$; MS (EI ion source): $m / z(\%)=377\left(26,\left[\mathrm{M}^{+}\right]\right), 348$ (77), 304 (100), 289 (14), 261 (18); HRMS: $m / z$ [M + Na] $]^{+}$calcd. for $\mathrm{C}_{20} \mathrm{H}_{18} \mathrm{~F}_{3} \mathrm{NO}_{3} \mathrm{Na}$ : 400.1131; found: 400.1119 .

Isomeric mixture $\mathbf{2 k}+\mathbf{2}^{\prime} \mathbf{k}$. Overall yield (catalyst A): $99 \%(107.2 .0 \mathrm{mg}) ; \mathbf{2 k} / \mathbf{2}^{\prime} \mathbf{k}=67 / 33$; overall yield (catalyst $\mathrm{A}^{\prime}$ ): $99 \%$ (107.1 mg); $\mathbf{2 k} / \mathbf{2}^{\prime} \mathbf{k}=94 / 6$; overall yield (catalyst B): $90 \%$

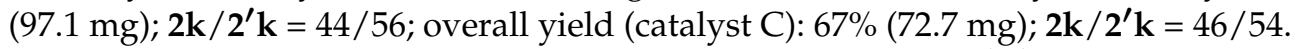

2k: orange oil; IR (Neat): 2980, 2243, 1705, 1596, $1494 \mathrm{~cm}^{-1} ;{ }^{1} \mathrm{H} \mathrm{NMR}(400.13 \mathrm{MHz})$ $\left(\mathrm{CDCl}_{3}\right): \delta=7.29-7.21(\mathrm{~m}, 6 \mathrm{H}), 6.90(\mathrm{~d}, J=8.7 \mathrm{~Hz}, 1 \mathrm{H}), 6.52\left(\mathrm{dd}, J_{1}=8.7 \mathrm{~Hz}, J_{2}=2.6 \mathrm{~Hz}\right.$, $1 \mathrm{H}), 5.80(\mathrm{t}, J=4.5 \mathrm{~Hz}, 1 \mathrm{H}), 4.38(\mathrm{~d}, J=4.6 \mathrm{~Hz}, 2 \mathrm{H}), 4.21(\mathrm{q}, J=7.1 \mathrm{~Hz}, 2 \mathrm{H}), 3.74(\mathrm{~s}, 3 \mathrm{H}), 1.27$ $(\mathrm{t}, J=7.1 \mathrm{~Hz}, 3 \mathrm{H}) ;{ }^{13} \mathrm{C} \mathrm{NMR}(100.6 \mathrm{MHz})\left(\mathrm{CDCl}_{3}\right): \delta=159.1(\mathrm{q}), 154.1(\mathrm{q}), 139.2(\mathrm{q}), 138.7$ (q), $138.6(\mathrm{q}), 128.8(\mathrm{CH}), 128.5(\mathrm{CH}), 127.8(\mathrm{CH}), 127.0(\mathrm{CH}), 122.5(\mathrm{q}), 120.4(\mathrm{CH}), 110.2$ $(\mathrm{CH}), 109.7(\mathrm{CH}), 62.3\left(\mathrm{CH}_{2}\right), 55.5\left(\mathrm{CH}_{3}\right), 43.3\left(\mathrm{CH}_{2}\right), 14.7\left(\mathrm{CH}_{3}\right)$. MS (EI ion source): $m / z$ $(\%)=309\left(0.2,\left[\mathrm{M}^{+}\right]\right), 235$ (100), 220 (17), 204 (29), 191 (24), 165 (15); HRMS: $m / z$ [M + Na] ${ }^{+}$ calcd. for $\mathrm{C}_{19} \mathrm{H}_{19} \mathrm{NO}_{3} \mathrm{Na}$ : 332.1257; found: 332.1260 .

$\mathbf{2}^{\prime} \mathbf{k}$ : colorless oil; IR (neat): 2982, 1708, 1610, 1504, $1466 \mathrm{~cm}^{-1} ;{ }^{1} \mathrm{H}$ NMR $(400.13 \mathrm{MHz})$ $\left(\mathrm{CDCl}_{3}\right): \delta=7.36-7.24(\mathrm{~m}, 6 \mathrm{H}), 6.69(\mathrm{~d}, J=8.9 \mathrm{~Hz}, 1 \mathrm{H}), 6.08(\mathrm{t}, J=5.1 \mathrm{~Hz}, 1 \mathrm{H}), 4.33(\mathrm{~d}$, $J=5.1 \mathrm{~Hz}, 2 \mathrm{H}), 4.29(\mathrm{q}, J=7.2 \mathrm{~Hz}, 2 \mathrm{H}), 3.43(\mathrm{~s}, 3 \mathrm{H}), 1.35(\mathrm{t}, J=7.2 \mathrm{~Hz}, 3 \mathrm{H}) ;{ }^{13} \mathrm{C} \mathrm{NMR}$ $(100.6 \mathrm{MHz})\left(\mathrm{CDCl}_{3}\right): \delta=156.2(\mathrm{q}), 153.9$ (q), 141.5 (q), 139.8 (q), 137.8 (q), $128.3(\mathrm{CH}), 127.7$ 
$(\mathrm{CH}), 126.68(\mathrm{CH}), 126.62(\mathrm{CH}), 124.5(\mathrm{CH}), 118.6(\mathrm{q}), 117.0(\mathrm{CH}), 108.4(\mathrm{CH}), 62.2\left(\mathrm{CH}_{2}\right)$, $55.5\left(\mathrm{CH}_{3}\right), 42.7\left(\mathrm{CH}_{2}\right), 14.7\left(\mathrm{CH}_{3}\right)$; $\mathrm{MS}$ (EI ion source): $m / z(\%)=309\left(42,\left[\mathrm{M}^{+}\right]\right), 280(51)$, 236 (100), 220 (51), 193 (15); HRMS: $m / z$ [M + Na ${ }^{+}$calcd. for $\mathrm{C}_{19} \mathrm{H}_{19} \mathrm{NO}_{3} \mathrm{Na}$ : 332.1257; found: 332.1260 .

Isomeric mixture $21+\mathbf{2}^{\prime} \mathbf{1}$. Overall yield (catalyst A): $98 \%(120.4 \mathrm{mg}) ; \mathbf{2 1} / \mathbf{2}^{\prime} \mathbf{1}=61 / 39$; overall yield (catalyst $\mathrm{A}^{\prime}$ ): $99 \%$ (120.9); $21 / \mathbf{2}^{\prime} \mathbf{1}=75 / 29$; overall yield (catalyst B): $99 \%$ (121.9 mg); 2l / 2'1 = 54/46; overall yield (catalyst C): 86\% (105.5 mg); 2l /2'1 = 54/46; yellow wax; IR (KBr): 3060, 2922, 1680, 1593, 1480, $1232 \mathrm{~cm}^{-1}$.

Reported NMR spectra refer to an isomeric mixture $21+2^{\prime} \mathbf{1}$ in the ratio $54 / 46 ;{ }^{1} \mathrm{H} \mathrm{NMR}$ signals were assigned to each specific isomer while ${ }^{13} \mathrm{C}$ NMR signals were not assigned.

${ }^{1} \mathrm{H}$ NMR $(400.13 \mathrm{MHz})\left(\mathrm{CDCl}_{3}\right)$ (selected signals): $\delta=7.89(\mathrm{~d}, J=8.4 \mathrm{~Hz}, 2 \mathrm{H} 2 \mathrm{l}), 7.81$ $\left(\mathrm{d}, J=8.4 \mathrm{~Hz}, 2 \mathrm{H} \mathrm{2} 2^{\prime} 1\right), 7.35(\mathrm{~d}, J=8.4 \mathrm{~Hz}, 2 \mathrm{H} 2 \mathrm{l}), 7.24-7.17\left(\mathrm{~m}, 1 \mathrm{H} 21+4 \mathrm{H} \mathrm{2}{ }^{\prime} 1\right), 6.84(\mathrm{~d}$, $J=8.6 \mathrm{~Hz}, 1 \mathrm{H} \mathrm{2l}), 6.59\left(\mathrm{~d}, J=9.0 \mathrm{~Hz}, 1 \mathrm{H} \mathrm{2} \mathbf{2}^{\prime} 1\right), 6.52\left(\mathrm{dd}, J_{1}=8.6 \mathrm{~Hz}, J_{2}=2.6 \mathrm{~Hz}, 1 \mathrm{H} 2 \mathrm{l}\right), 6.03$ $\left(\mathrm{t}, J=5.0 \mathrm{~Hz}, 1 \mathrm{H} \mathrm{2} \mathbf{2}^{\prime} 1\right), 5.87(\mathrm{t}, J=4.6 \mathrm{~Hz}, 1 \mathrm{H} \mathrm{2l}), 4.39(\mathrm{~d}, J=4.6 \mathrm{~Hz}, 2 \mathrm{H} \mathrm{2l}), 4.25-4.15(\mathrm{~m}$, $\left.2 \mathrm{H} 2 \mathrm{l}+4 \mathrm{H} \mathrm{2} \mathbf{2}^{\prime} 1\right), 3.74$ (s, 3H 2l), 3.32 (s, 3H 2'1), 2.54 (s, 3H 21), 2.53 (s, 3H 2'1), 1.29-1.23 (m, $3 \mathrm{H} 21$ + 3H 2' 1$) ;{ }^{13} \mathrm{C}$ NMR (100.6 MHz) $\left(\mathrm{CDCl}_{3}\right): \delta=198.1,197.9,159.4,156.0,154.1,153.9$, 146.7, 144.1, 139.8, 138.7, 138.1, 137.1, 136.6, 135.5, 129.0, 128.8, 128.7, 128.0, 126.93, 126.90, $126.0,121.9,121.7,117.9,117.1,110.4,109.9,108.2,62.43,62.39,55.6,55.4,43.2,42.7,26.85$, 26.79, 14.8, 14.7; MS (EI ion source): $m / z(\%)=351\left(51,\left[\mathrm{M}^{+}\right]\right), 322(63), 278(100), 262$ (23), 235 (20), 43 (23); HRMS: $m / z[\mathrm{M}+\mathrm{H}]^{+}$calcd. for $\mathrm{C}_{21} \mathrm{H}_{22} \mathrm{NO}_{4}$ : 352.1543; found: 352.1536 .

Isomeric mixture $\mathbf{2 m}+\mathbf{2}^{\prime} \mathbf{m}$. Overall yield (catalyst A): $70 \%(83.0 \mathrm{mg}) ; \mathbf{2} \mathbf{m} / \mathbf{2}^{\prime} \mathbf{m}=91 / 9$; overall yield (catalyst $\mathrm{A}^{\prime}$ ): $73 \%(86.5 \mathrm{mg}) ; \mathbf{2 m} / \mathbf{2}^{\prime} \mathrm{m}=91 / 9$; overall yield (catalyst B): $83 \%$ (98.6 mg); $\mathbf{2 m} / \mathbf{2}^{\prime} \mathbf{m}=63 / 37$; overall yield (catalyst C): $77 \%(91.8 \mathrm{mg}) ; \mathbf{2 m} / \mathbf{2}^{\prime} \mathbf{m}=51 / 49$.

Compound 2m. Colorless oil; IR (Neat): 2915, 1711, 1577, 1386, $1244 \mathrm{~cm}^{-1} ;{ }^{1} \mathrm{H}$ NMR $(400.13 \mathrm{MHz})\left(\mathrm{CDCl}_{3}\right): \delta=7.27-7.24(\mathrm{~m}, 3 \mathrm{H}), 7.00(\mathrm{~d}, J=8.6 \mathrm{~Hz}, 1 \mathrm{H}), 6.91(\mathrm{~d}, J=8.8 \mathrm{~Hz}$, $2 \mathrm{H}), 6.60\left(\mathrm{dd}, J_{1}=8.6 \mathrm{~Hz}, J_{2}=2.6 \mathrm{~Hz}, 1 \mathrm{H}\right), 5.84(\mathrm{t}, J=4.5 \mathrm{~Hz}, 1 \mathrm{H}), 4.43(\mathrm{~d}, J=4.5 \mathrm{~Hz}$, $2 \mathrm{H}), 4.28(\mathrm{q}, J=7.1 \mathrm{~Hz}, 2 \mathrm{H}), 3.84(\mathrm{~s}, 3 \mathrm{H}), 3.82(\mathrm{~s}, 3 \mathrm{H}), 1.35(\mathrm{t}, J=7.1 \mathrm{~Hz}, 3 \mathrm{H}) ;{ }^{13} \mathrm{C} \mathrm{NMR}$ $(100.6 \mathrm{MHz})\left(\mathrm{CDCl}_{3}\right): \delta=159.3(\mathrm{q}), 159.0(\mathrm{q}), 154.1(\mathrm{q}), 138.6(\mathrm{q}), 138.2(\mathrm{q}), 131.6(\mathrm{q}), 129.9$ $(\mathrm{CH}), 127.0(\mathrm{CH}), 122.7(\mathrm{q}), 119.6(\mathrm{CH}), 113.9(\mathrm{CH}), 110.2(\mathrm{CH}), 109.6(\mathrm{CH}), 62.2\left(\mathrm{CH}_{2}\right), 55.5$ $\left(\mathrm{CH}_{3}\right), 55.4\left(\mathrm{CH}_{3}\right), 43.2\left(\mathrm{CH}_{2}\right), 14.7\left(\mathrm{CH}_{3}\right)$; $\mathrm{MS}$ (EI ion source): $m / z(\%)=339\left(1,\left[\mathrm{M}^{+}\right]\right), 265$ (100), 250 (13), 222 (10), 207 (15); HRMS: $m / z[\mathrm{M}+\mathrm{K}]^{+}$calcd. for $\mathrm{C}_{20} \mathrm{H}_{21} \mathrm{NO}_{4} \mathrm{~K}: 378.1102$; found: 378.1095 .

Compound 2' m. Colorless oil; IR (neat): 2915, 1694, 1609, 1381, 1239, $1042 \mathrm{~cm}^{-1}$; ${ }^{1} \mathrm{H} \mathrm{NMR}(400.13 \mathrm{MHz})\left(\mathrm{CDCl}_{3}\right): \delta=7.26-7.24(\mathrm{~m}, 1 \mathrm{H}), 7.17(\mathrm{t}, J=8.2 \mathrm{~Hz}, 1 \mathrm{H}), 7.08(\mathrm{~d}$, $J=8.8 \mathrm{~Hz}, 2 \mathrm{H}), 6.75(\mathrm{~d}, J=8.8 \mathrm{~Hz}, 2 \mathrm{H}), 6.60(\mathrm{~d}, J=8.9 \mathrm{~Hz}, 1 \mathrm{H}), 5.93(\mathrm{t}, J=5.1 \mathrm{~Hz}, 1 \mathrm{H}), 4.20$ $(\mathrm{d}, J=5.1 \mathrm{~Hz}, 2 \mathrm{H}), 4.18(\mathrm{q}, J=7.1 \mathrm{~Hz}, 2 \mathrm{H}), 3.75(\mathrm{~s}, 3 \mathrm{H}), 3.37(\mathrm{~s}, 3 \mathrm{H}), 1.25(\mathrm{t}, J=7.1 \mathrm{~Hz}, 3 \mathrm{H})$; ${ }^{13} \mathrm{C}$ NMR (100.6 MHz) $\left(\mathrm{CDCl}_{3}\right): \delta=158.6(\mathrm{q}), 156.3(\mathrm{q}), 153.9(\mathrm{q}), 140.0(\mathrm{q}), 137.2(\mathrm{q}), 134.1$ (q), $128.3(\mathrm{CH}), 127.8(\mathrm{CH}), 123.6(\mathrm{CH}), 118.7(\mathrm{q}), 117.0(\mathrm{CH}), 113.1(\mathrm{CH}), 108.4(\mathrm{CH}), 62.3$ $\left(\mathrm{CH}_{2}\right), 55.7\left(\mathrm{CH}_{3}\right), 55.5\left(\mathrm{CH}_{3}\right), 42.7\left(\mathrm{CH}_{2}\right), 14.7\left(\mathrm{CH}_{3}\right)$; $\mathrm{MS}$ (EI ion source): $m / z(\%)=339(66$, $\left.\left[\mathrm{M}^{+}\right]\right), 310$ (67), 266 (100), 251 (23); HRMS: $m / z[\mathrm{M}+\mathrm{H}]^{+}$calcd. for $\mathrm{C}_{20} \mathrm{H}_{22} \mathrm{NO}_{4}$ : 340.1543; found: 340.1539 .

Isomeric mixture $\mathbf{2 n}+\mathbf{2}^{\prime} \mathbf{n}$. Overall yield (catalyst A): $85 \%(112.9 \mathrm{mg}) ; \mathbf{2 n} / \mathbf{2}^{\prime} \mathbf{n}=88 / 12$; overall yield (catalyst $A^{\prime}$ ): $75 \%$ (99.7); $2 \mathbf{n} / \mathbf{2}^{\prime} \mathbf{n}=64 / 36$; overall yield (catalyst B): $99 \%$ (131.6 mg); 2n/2'n = 40/60; overall yield (catalyst C): 72\% (95.1 mg); $\mathbf{2 n / 2} \mathbf{n}=19 / 81$.

Compound 2n: pale yellow solid; m.p. = 140-141 ${ }^{\circ} \mathrm{C}$; IR (neat): 2930, 1751, 1657, 1583, $1298 \mathrm{~cm}^{-1} ;{ }^{1} \mathrm{H}$ NMR $(400.13 \mathrm{MHz})\left(\mathrm{CDCl}_{3}\right): \delta=8.27(\mathrm{bs}, 1 \mathrm{H}), 8.08(\mathrm{~d}, J=8.4 \mathrm{~Hz}, 2 \mathrm{H}), 7.62$ $\left(\mathrm{dd}, J_{1}=8.2 \mathrm{~Hz}, J_{2}=1.7 \mathrm{~Hz}, 1 \mathrm{H}\right), 7.41(\mathrm{~d}, J=8.4 \mathrm{~Hz}, 2 \mathrm{H}), 7.08(\mathrm{~d}, J=8.2 \mathrm{~Hz}, 1 \mathrm{H}), 6.21(\mathrm{t}$, $J=4.5 \mathrm{~Hz}, 1 \mathrm{H}), 4.53(\mathrm{~d}, J=4.5 \mathrm{~Hz}, 2 \mathrm{H}), 4.31(\mathrm{q}, J=7.1 \mathrm{~Hz}, 2 \mathrm{H}), 3.94(\mathrm{~s}, 3 \mathrm{H}), 2.60(\mathrm{~s}, 3 \mathrm{H})$, $1.37(\mathrm{t}, J=7.1 \mathrm{~Hz}, 3 \mathrm{H}) ;{ }^{13} \mathrm{C} \mathrm{NMR}(100.6 \mathrm{MHz})\left(\mathrm{CDCl}_{3}\right): \delta=197.3(\mathrm{q}), 166.7(\mathrm{q}), 153.9$ (q), $142.8(\mathrm{q}), 137.7(\mathrm{q}), 137.3(\mathrm{q}), 136.4(\mathrm{q}), 132.6,(\mathrm{q}) 129.9(\mathrm{CH}), 129.8(\mathrm{CH}), 128.7(\mathrm{CH}), 126.9$ (q), $125.9(\mathrm{CH}), 124.4(\mathrm{CH}), 123.9(\mathrm{CH}), 62.5\left(\mathrm{CH}_{2}\right), 52.2\left(\mathrm{CH}_{3}\right), 43.0\left(\mathrm{CH}_{2}\right), 26.7\left(\mathrm{CH}_{3}\right), 14.5$ $\left(\mathrm{CH}_{3}\right)$; MS (EI ion source): $m / z(\%)=379$ (67, [M $\left.\left.{ }^{+}\right]\right), 350$ (15), 306 (100), 290 (52), 264 (70), 204 (26); HRMS: $m / z$ [M + Na] ${ }^{+}$calcd. for $\mathrm{C}_{22} \mathrm{H}_{21} \mathrm{NO}_{5} \mathrm{Na}$ : 402.1312; found: 402.1312. 
Compound 2'n: yellow oil; IR (neat): 2919, 1725, 1599, 1268, $1023 \mathrm{~cm}^{-1} ;{ }^{1} \mathrm{H}$ NMR $(400.13 \mathrm{MHz})\left(\mathrm{CDCl}_{3}\right): \delta=7.90(\mathrm{~d}, J=8.5 \mathrm{~Hz}, 2 \mathrm{H}), 7.77(\mathrm{~m}, 1 \mathrm{H}), 7.30(\mathrm{t}, J=7.8 \mathrm{~Hz}, 1 \mathrm{H})$, $7.23-7.19(\mathrm{~m}, 3 \mathrm{H}), 6.21(\mathrm{t}, J=5.2 \mathrm{~Hz}, 1 \mathrm{H}), 4.33(\mathrm{~d}, J=5.2 \mathrm{~Hz}, 2 \mathrm{H}), 4.21(\mathrm{q}, J=7.1 \mathrm{~Hz}, 2 \mathrm{H}), 3.84$ $(\mathrm{s}, 3 \mathrm{H}), 2.00(\mathrm{~s}, 3 \mathrm{H}), 1.27(\mathrm{t}, J=7.1 \mathrm{~Hz}, 3 \mathrm{H}) ;{ }^{13} \mathrm{C} \mathrm{NMR}(100.6 \mathrm{MHz})\left(\mathrm{CDCl}_{3}\right): \delta=202.2(\mathrm{q})$, 166.7 (q), $153.6(\mathrm{q}), 145.4(\mathrm{q}), 139.8(\mathrm{q}), 139.5(\mathrm{q}), 138.0(\mathrm{q}), 129.9(\mathrm{q}), 129.1(\mathrm{CH}), 127.6(\mathrm{CH})$, 127.3 (CH), 127.1 (q), $126.7(\mathrm{CH}), 123.9(\mathrm{CH}), 62.5\left(\mathrm{CH}_{2}\right), 52.1\left(\mathrm{CH}_{3}\right), 42.4\left(\mathrm{CH}_{2}\right), 29.1\left(\mathrm{CH}_{3}\right)$, $14.5\left(\mathrm{CH}_{3}\right)$; MS (EI ion source): $\mathrm{m} / z(\%)=379\left(58,\left[\mathrm{M}^{+}\right]\right), 350$ (100), 306 (95), 264 (44), 204 (36), 43 (27); HRMS: $m / z$ [M + Na] ${ }^{+}$calcd. for $\mathrm{C}_{22} \mathrm{H}_{21} \mathrm{NO}_{5} \mathrm{Na}$ : 402.1312 ; found: 402.1310.

Isomeric mixture $\mathbf{2 o}+\mathbf{2}^{\prime} \mathbf{o}$. Overall yield (catalyst $\left.\mathrm{A}\right): 88 \%(112.0 \mathrm{mg}) ; \mathbf{2 o} / \mathbf{2}^{\prime} \mathbf{o}=88 / \mathbf{1 2}$; overall yield (catalyst $A^{\prime}$ ): $90 \%$ (114.3); $\mathbf{2 o} / \mathbf{2}^{\prime} \mathbf{o}=65 / 35$; overall yield (catalyst B): $70 \%$ $(89.0 \mathrm{mg}) ; \mathbf{2 o} / \mathbf{2}^{\prime} \mathbf{o}=33 / 67 ;$ overall yield (catalyst C): $82 \%(104.4 \mathrm{mg}) ; \mathbf{2 0} / \mathbf{2}^{\prime} \mathbf{o}=20 / 80$.

Compound 2o. Yellow wax; IR (neat): 2982, 1680, 1607, 1556, $1256 \mathrm{~cm}^{-1} ;{ }^{1} \mathrm{H}$ NMR $(400.13 \mathrm{MHz})\left(\mathrm{CDCl}_{3}\right): \delta=8.27(\mathrm{bs}, 1 \mathrm{H}), 8.00(\mathrm{~d}, J=8.4 \mathrm{~Hz}, 2 \mathrm{H}), 7.63\left(\mathrm{dd}, J_{1}=8.2 \mathrm{~Hz}\right.$, $\left.J_{2}=1.7 \mathrm{~Hz}, 1 \mathrm{H}\right), 7.43(\mathrm{~d}, J=8.4 \mathrm{~Hz}, 2 \mathrm{H}), 7.08(\mathrm{~d}, J=8.2 \mathrm{~Hz}, 1 \mathrm{H}), 6.22(\mathrm{t}, J=4.6 \mathrm{~Hz}, 1 \mathrm{H})$, $4.54(\mathrm{~d}, J=4.6 \mathrm{~Hz}, 2 \mathrm{H}), 4.32(\mathrm{q}, J=7.1 \mathrm{~Hz}, 2 \mathrm{H}), 2.64(\mathrm{~s}, 3 \mathrm{H}), 2.60(\mathrm{~s}, 3 \mathrm{H}), 1.37(\mathrm{t}, J=7.1 \mathrm{~Hz}$, $3 \mathrm{H}) ;{ }^{13} \mathrm{C}$ NMR (100.6 MHz) $\left(\mathrm{CDCl}_{3}\right): \delta=197.8(\mathrm{q}), 197.5(\mathrm{q}), 154.0(\mathrm{q}), 143.1(\mathrm{q}), 137.9(\mathrm{q})$, $137.5(\mathrm{q}), 136.9(\mathrm{q}), 136.7(\mathrm{q}), 132.7(\mathrm{q}), 129.1(\mathrm{CH}), 128.9(\mathrm{CH}), 127.2(\mathrm{CH}), 126.1(\mathrm{CH}), 124.6$ $(\mathrm{CH}), 124.0(\mathrm{CH}), 62.7\left(\mathrm{CH}_{2}\right), 43.3\left(\mathrm{CH}_{2}\right), 26.9\left(\mathrm{CH}_{3}, 2 \mathrm{C}\right), 14.7\left(\mathrm{CH}_{3}\right)$; MS (EI ion source): $m / z(\%)=363\left(62,\left[\mathrm{M}^{+}\right]\right), 333(12), 290(83), 248(70), 43$ (100); HRMS: $m / z[\mathrm{M}+\mathrm{Na}]^{+}$calcd. for $\mathrm{C}_{22} \mathrm{H}_{21} \mathrm{NO}_{4} \mathrm{Na}$ : 386.1363; found: 386.1359 .

Compound 2'o. Yellow wax; IR (neat): 2981, 1682, 1603, 1450, 1376, $1265 \mathrm{~cm}^{-1}$; ${ }^{1} \mathrm{H}$ NMR $(400.13 \mathrm{MHz})\left(\mathrm{CDCl}_{3}\right): \delta=7.89(\mathrm{~d}, J=8.6 \mathrm{~Hz}, 2 \mathrm{H}), 7.85(\mathrm{~m}, 1 \mathrm{H}) 7.38(\mathrm{t}, J=7.7 \mathrm{~Hz}$, $1 \mathrm{H}), 7.31-7.27(\mathrm{~m}, 3 \mathrm{H}), 6.28(\mathrm{t}, J=5.2 \mathrm{~Hz}, 1 \mathrm{H}), 4.40(\mathrm{~d}, J=5.2 \mathrm{~Hz}, 2 \mathrm{H}), 4.28(\mathrm{q}, J=7.1 \mathrm{~Hz}, 2 \mathrm{H})$, $2.59(\mathrm{~s}, 3 \mathrm{H}), 2.07(\mathrm{~s}, 3 \mathrm{H}), 1.34(\mathrm{t}, J=7.1 \mathrm{~Hz}, 3 \mathrm{H}) ;{ }^{13} \mathrm{C} \mathrm{NMR}(100.6 \mathrm{MHz})\left(\mathrm{CDCl}_{3}\right): \delta=202.3$ (q), 197.6 (q), 153.7 (q), 145.8 (q), 139.9 (q), 139.6 (q), 138.1 (q), 136.1 (q), $128.8(\mathrm{CH}), 127.7$ $(\mathrm{CH}), 127.39(\mathrm{CH}), 127.34(\mathrm{q}), 126.9(\mathrm{CH}), 124.1(\mathrm{CH}), 62.6\left(\mathrm{CH}_{2}\right), 42.5\left(\mathrm{CH}_{2}\right), 29.3\left(\mathrm{CH}_{3}\right)$, $26.7\left(\mathrm{CH}_{3}\right), 14.6\left(\mathrm{CH}_{3}\right)$; MS (EI ion source): $m / z(\%)=363\left(26,\left[\mathrm{M}^{+}\right]\right), 334(41), 290(54)$, 232 (28), 204 (30), 43 (100); HRMS: $m / z$ [M + Na] ${ }^{+}$calcd. for $\mathrm{C}_{22} \mathrm{H}_{21} \mathrm{NO}_{4} \mathrm{Na}$ : 386.1363; found: 386.1361 .

\subsubsection{Characterization Data of Compounds $\mathbf{3 a}, \mathbf{5 b}, \mathbf{6 b}, \mathbf{7 b}$}

Characterization data of the listed compounds are reported in the Supplementary Materials.

\section{Conclusions}

The employment of ethyl carbamate $\mathrm{N}$-protecting group represents a viable alternative to the tosyl $\mathrm{N}$-protecting group, allowing the efficient synthesis of the corresponding 4-substituted-1,2-dihydroquinolines by means of the gold-catalyzed IMHA reaction. The reaction proceeds with internal alkynes bearing electron-rich and electron-deficient substituents in the benzenes affording only the 6-endo cyclization product in fair to high yields. $\mathrm{Au}(\mathrm{I})$ - catalyzed regiodivergent intramolecular hydroarylation of the $\mathrm{N}$-ethoxycarbonyl$\mathrm{N}$-propargyl-meta-substituted anilines at the ortho- and para-position cyclization could be successfully established respectively through fine-tuning electronic and steric effects of the gold complexes ligands.

Supplementary Materials: The following are available online, general information of reagents and methods, synthetic procedures, and characterization data.

Author Contributions: Project administration, G.F.; Supervision, A.G.; Conceptualization, A.G. and A.I.; Methodology, A.G. and A.I.; Investigation, A.F., F.M., A.S. and G.M.; Data curation, A.I.; Writing — original draft preparation, review and editing, A.A.; Writing — review and editing, V.M., A.C., A.G. and A.I. All authors have read and agreed to the published version of the manuscript.

Funding: This research received no external funding.

Institutional Review Board Statement: Not applicable. 
Informed Consent Statement: Not applicable.

Data Availability Statement: Data is contained within the article or supplementary material.

Acknowledgments: We gratefully acknowledge the PRIN project 2017 “Targeting Hedgehog pathway: virtual screening identification and sustainable synthesis of novel Smo and Gli inhibitors and their pharmacological drug delivery strategies for improved therapeutic effects in tumors." (20175XBSX4) and La Sapienza, University of Rome, for financial support.

Conflicts of Interest: The authors declare no conflict of interest.

Sample Availability: Samples of the compounds are not available from the authors.

\section{References}

1. Vessally, E.; Edjlali, L.; Hosseinian, A.; Bekhradnia, A.; Esrafili, M.D. Novel routes to quinoline derivatives from Npropargylamines. Rsc. Adv. 2016, 6, 49730-49746. [CrossRef]

2. Hussain, H.; Al-Harrasi, A.; Al-Rawahi, A.; Green, I.R.; Gibbons, S. Fruitful Decade for Antileishmanial Compounds from 2002 to Late 2011. Chem. Rev. 2014, 114, 10369-10428. [CrossRef]

3. Pratap, R.; Ram, V.J. Natural and Synthetic Chromenes, Fused Chromenes, and Versatility of Dihydrobenzo[h]chromenes in Organic Synthesis. Chem. Rev. 2014, 114, 10476-10526. [CrossRef]

4. Bhanja, C.; Jena, S.; Nayak, S.; Mohapatra, S. Organocatalytic tandem Michael addition reactions: A powerful access to the enantioselective synthesis of functionalized chromenes, thiochromenes and 1,2-dihydroquinolines. Beilstein J. Org. Chem 2012, 8 , 1668-1694. [CrossRef]

5. Barluenga, J.; Rodriguez, F.; Fananas, F.J. Recent Advances in the Synthesis of Indole and Quinoline Derivatives through Cascade Reactions. Chem-Asian J. 2009, 4, 1036-1048. [CrossRef]

6. Amrutham, V.; Radikovich, A.M.; Mameda, N.; Gajula, K.S.; Grigor'eva, N.G.; Ivanovich, K.B.; Akula, V.; Nama, N. A heterogeneous catalytic and solvent-free approach to 1,2-dihydroquinoline derivatives from aromatic amines and alkynes by tandem hydroarylation-hydroamination. Catal. Commun. 2020, 135, 105888. [CrossRef]

7. Pucheta, A.; Mendieta, A.; Madrigal, D.A.; Hernández-Benitez, R.I.; Romero, L.; Garduño-Siciliano, L.; Rugerio-Escalona, C.; Cruz-López, M.C.; Jiménez, F.; Ramírez-Villalva, A.; et al. Synthesis and biological activity of fibrate-based acyl- and alkyl-phenoxyacetic methyl esters and 1,2-dihydroquinolines. Med. Chem. Res. 2020, 29, 459-478. [CrossRef]

8. Das, R.; Khot, N.P.; Deshpande, A.S.; Kapur, M. Catalyst Control in Switching the Site Selectivity of C-H Olefinations of 1,2-Dihydroquinolines: An Approach to Positional-Selective Functionalization of Quinolines. Chem. Eur. J. 2020, 26, 927-938. [CrossRef]

9. El-Harairy, A.; Yiliqi; Lai, B.; Vaccaro, L.; Li, M.; Gu, Y. A Sulfone-Containing Imidazolium-Based Brønsted Acid Ionic Liquid Catalyst Enables Replacing Dipolar Aprotic Solvents with Butyl Acetate. Adv. Synth. Catal. 2019, 361, 3342-3350. [CrossRef]

10. Wu, S.; Liu, C.; Luo, G.; Jin, Z.; Zheng, P.; Chi, Y.R. NHC-Catalyzed Chemoselective Reactions of Enals and Aminobenzaldehydes for Access to Chiral Dihydroquinolines. Angew. Chem. Int. Edit. 2019, 58, 18410-18413. [CrossRef]

11. El-Remaily, M.A.E.A.A.A.; Abu-Dief, A.M.; Elhady, O. Green synthesis of TiO2 nanoparticles as an efficient heterogeneous catalyst with high reusability for synthesis of 1,2-dihydroquinoline derivatives. Appl. Organomet. Chem. 2019, 33, e5005. [CrossRef]

12. Goulart, T.A.C.; Kazmirski, J.A.G.; Back, D.F.; Zeni, G. Iron(III)-Promoted Synthesis of 3-(Organoselanyl)-1,2-Dihydroquinolines from Diorganyl Diselenides and N-Arylpropargylamines by Sequential Carbon-Carbon and Carbon-Selenium Bond Formation. Adv. Synth. Catal. 2019, 361, 96-104. [CrossRef]

13. Nevado, C.; Echavarren, A.M. Intramolecular Hydroarylation of Alkynes Catalyzed by Platinum or Gold: Mechanism and endo Selectivity. Chem. Eur. J. 2005, 11, 3155-3164. [CrossRef] [PubMed]

14. Johannsen, T.; Golz, C.; Alcarazo, M. $\alpha$-Cationic Phospholes: Synthesis and Applications as Ancillary Ligands. Angew. Chem. Int. Ed. 2020, 59, 22779-22784. [CrossRef] [PubMed]

15. Ding, D.; Mou, T.; Feng, M.; Jiang, X. Utility of Ligand Effect in Homogenous Gold Catalysis: Enabling Regiodivergent $\pi$-Bond-Activated Cyclization. J. Am. Chem. Soc. 2016, 138, 5218-5221. [CrossRef]

16. Menon, R.S.; Findlay, A.D.; Bissember, A.C.; Banwell, M.G. The Au(I)-Catalyzed Intramolecular Hydroarylation of Terminal Alkynes Under Mild Conditions: Application to the Synthesis of $2 \mathrm{H}-\mathrm{Chromenes,} \mathrm{Coumarins,} \mathrm{Benzofurans,} \mathrm{and} \mathrm{Dihydroquino-}$ lines. J. Org. Chem. 2009, 74, 8901-8903. [CrossRef] [PubMed]

17. Alonso-Marañón, L.; Sarandeses, L.A.; Martínez, M.M.; Pérez Sestelo, J. Synthesis of fused chromenes by the indium(iii)-catalyzed cascade hydroarylation/cycloisomerization reactions of polyyne-type aryl propargyl ethers. Org. Chem. Front. 2018, 5, $2308-2312$. [CrossRef]

18. Alonso-Marañón, L.; Sarandeses, L.A.; Martínez, M.M.; Pérez Sestelo, J. Sequential In-catalyzed intramolecular hydroarylation and Pd-catalyzed cross-coupling reactions using bromopropargyl aryl ethers and amines. Org. Chem. Front. 2017, 4, 500-505. [CrossRef]

19. Zhang, X.; Larock, R.C. Synthesis of Spiro[4.5]trienones by Intramolecular ipso-Halocyclization of 4-(p-Methoxyaryl)-1-alkynes. J. Am. Chem. Soc. 2005, 127, 12230-12231. [CrossRef] 
20. Arcadi, A.; Fabrizi, G.; Fochetti, A.; Franzini, R.; Ghirga, F.; Goggiamani, A.; Iazzetti, A.; Marrone, F.; Serraiocco, A. Synthesis of Polycyclic Chromene Cores through Gold (I)-Catalyzed Intramolecular Hydroarylation Reaction (IMHA). Eur. J. Org. Chem. 2021, 1676-1687. [CrossRef]

21. Arcadi, A.; Ciogli, A.; Fabrizi, G.; Fochetti, A.; Franzini, R.; Ghirga, F.; Goggiamani, A.; Iazzetti, A. Synthesis of pyrano[2,3f]chromen-2-ones vs. pyrano[3,2-g]chromen-2-ones through site controlled gold-catalyzed annulations. Org. Biomol Chem 2019, 17, 10065-10072. [CrossRef]

22. Arcadi, A.; Blesi, F.; Cacchi, S.; Fabrizi, G.; Goggiamani, A.; Marinelli, F. Gold versus silver catalyzed intramolecular hydroarylation reactions of [(3-arylprop-2-ynyl)oxy]benzene derivatives. Org. Biomol Chem 2012, 10, 9700-9708. [CrossRef]

23. Ghosh, A.K.; Brindisi, M. Organic Carbamates in Drug Design and Medicinal Chemistry. J. Med. Chem. 2015, 58, 2895-2940. [CrossRef]

24. Alfonsi, M.; Arcadi, A.; Chiarini, M.; Marinelli, F. Sequential alkylation/gold-catalyzed annulation reactions of anilines with propargylic bromide derivatives. Tetrahedron Lett. 2011, 52, 5145-5148. [CrossRef]

25. Arcadi, A.; Alfonsi, M.; Chiarini, M.; Marinelli, F. Sequential gold-catalyzed reactions of 1-phenylprop-2-yn-1-ol with 1,3dicarbonyl compounds. J. Organomet. Chem. 2009, 694, 576-582. [CrossRef]

26. Martín-Matute, B.; Nevado, C.; Cárdenas, D.J.; Echavarren, A.M. Intramolecular Reactions of Alkynes with Furans and Electron Rich Arenes Catalyzed by $\mathrm{PtCl}_{2}$ : The Role of Platinum Carbenes as Intermediates. J. Am. Chem. Soc. 2003, 125, 5757-5766. [CrossRef]

27. Abbiati, G.; Arcadi, A.; Bianchi, G.; Di Giuseppe, S.; Marinelli, F.; Rossi, E. Sequential Amination/Annulation/Aromatization Reaction of Carbonyl Compounds and Propargylamine: A New One-Pot Approach to Functionalized Pyridines. J. Org. Chem. 2003, 68, 6959-6966. [CrossRef]

28. Chintawar, C.C.; Yadav, A.K.; Kumar, A.; Sancheti, S.P.; Patil, N.T. Divergent Gold Catalysis: Unlocking Molecular Diversity through Catalyst Control. Chem. Rev. 2021. [CrossRef] [PubMed]

29. Yang, Y.; Liu, Y.; Lv, P.; Zhu, R.; Liu, C.; Zhang, D. Theoretical Insight into the Mechansim and Origin of Ligand-Controlled Regioselectivity in Homogenous Gold-Catalyzed Intramolecular Hydroarylation of Alkynes. J. Org. Chem. 2018, 83, $2763-2772$. [CrossRef]

30. Vacala, T.; Bejcek, L.P.; Williams, G.; Williamson, A.C.; Vadola, P.A. Gold-Catalyzed Hydroarylation of N-Aryl Alkynamides for the Synthesis of 2-Quinolinones. J. Org. Chem. 2017, 82, 2558-2569. [CrossRef] 